\title{
Incidence of Childhood Obesity in the United States
}

\author{
Solveig A. Cunningham, Ph.D., Michael R. Kramer, Ph.D., K.M. Venkat Narayan, M.D. \\ Emory University, Atlanta, GA
}

\section{The Authors Reply:}

Childhood obesity is complex, and multiple approaches are needed to identify vulnerable children. Although we agree with Kuller that quantifying growth trajectories may be informative, doing so is less practical as a screening tool for assessing the future risk of obesity than determining weight status at the age of 5 years because trajectories require multiple points of measurement. Our principal finding was that new cases of obesity are most frequent at the youngest ages. This finding, which offers new insight into windows for prevention, complements the oft-reported data on cross-sectional prevalence.

Boelsen-Robinson and colleagues emphasize another complexity of childhood obesity important differences in the prevalence of obesity across socioeconomic groups. We would like to highlight three patterns that emerge from studying incidence.

First, to understand patterns of obesity related to socioeconomic status, a comparison of the wealthiest families with the poorest families is not sufficient, since a child's weight in kindergarten matters across the socioeconomic spectrum. We did not find a linear socioeconomic gradient in the incidence of obesity (Fig. 1). In the entire cohort, the incidence of obesity from 5 through 14 years of age was significantly lower among children from the wealthiest $20 \%$ of families than among all other children. The risks of obesity were relatively flat among children in the poorer socioeconomic quintiles; the greatest risk of becoming obese was among children from middle-income families, and there was some attenuation in the incidence of obesity only among children in the second wealthiest quintile.

Second, the risk of obesity was higher from 5 through 14 years of age across socioeconomic quintiles among children who had been overweight as kindergartners than among those who had had a normal weight in kindergarten. Indeed, the risk of obesity among children from the poorer $60 \%$ of families was double the risk among children from the wealthiest $20 \%$. Still, even among children from the wealthiest quintile, the risk of becoming obese was 5 times higher among children who had been overweight in kindergarten than among children who had had a normal weight in kindergarten; among children from the poorest quintile, the risk of obesity was approximately 3.4 times higher among those who had been overweight in kindergarten than among those who had had a normal weight in kindergarten.

sargese@emory.edu.

Since publication of their article, the authors report no further potential conflict of interest. 
Third, socioeconomic status is a stronger predictor of obesity among normal-weight children than among overweight children. The relative risk of becoming obese among children with lower socioeconomic status as compared with children with higher socioeconomic status was greater for normal-weight kindergartners than for overweight kindergartners.

The patterns of the incidence of obesity during childhood indicate the complexities of the socioeconomic context of this health problem and the reach of the risk of obesity across the socioeconomic spectrum in the United States. 




\section{Quintile of Family Socioeconomic Status in Kindergarten}

Figure 1. Cumulative Incidence of Obesity from Kindergarten through 14 Years of Age.

The cumulative incidence of obesity according to the quintile of socioeconomic status in kindergarten and the weight status (overweight or normal weight) in kindergarten among children in the United States is shown. The I bars represent 95\% confidence intervals. 